\title{
AN INTRODUCTION TO FULLY AUGMENTED LINKS
}

\author{
JESSICA S. PURCELL
}

\begin{abstract}
In this article we summarize information on the class of fully augmented links. These links are geometrically explicit, and therefore provide a large class of examples of hyperbolic links for which geometric information can be computed.
\end{abstract}

\section{INTRODUCTION}

The purpose of this paper is primarily expository, to introduce students and researchers in 3-manifolds, hyperbolic geometry, knot theory, and related fields to a class of hyperbolic links which is geometrically explicit, but seems to be underutilized.

The class which we study is that of fully augmented links. These are obtained from diagrams of links in $S^{3}$ as follows. Let $K$ be a link with diagram $D(K)$. Regard $D(K)$ as a 4-valent graph in the plane. A string of bigon regions of the complement of this graph arranged end to end is called a twist region, as is a vertex adjacent to no bigons. We assume throughout that the diagram is alternating in a twist region, else it can be replaced by a diagram with fewer crossings in an obvious way. To form a fully augmented link, we encircle each twist region with a single unknotted component, called a crossing circle. The complement of the result is homeomorphic to the link obtained by removing all full-twists, i.e. pairs of crossings, from each twist region. See Figure 1. A diagram of the fully augmented link therefore contains a finite number of crossing circle components, each perpendicular to the projection plane and encircling exactly two strands of the link. The other link components are embedded on the projection plane, except possibly for a finite number of single crossings adjacent to crossing circles. These single crossings we call half-twists.

The original link complement can be obtained from the fully augmented link by performing $(p, 1)$ Dehn filling on the crossing circles, for an appropriate choice of $p$. This process appears, for example, in Rolfsen's book [15].

Geometric properties of augmented links seem to have been studied first by Adams [2]. Rather than adding a crossing circle to each twist region, or augmenting, Adams considered diagrams where some collection of twist regions are augmented. He showed that if the original link has a nonsplit prime alternating diagram, and is not a $(2, q)$ torus link, then any augmentation is a hyperbolic link.

These ideas were used by Lackenby to show that families of alternating knots admit no exceptional Dehn fillings [8], and to determine information on the volumes of alternating knots [9]. In an appendix to [9], I. Agol and D. Thurston investigated the geometry of fully augmented links, using a decomposition of the link complement into ideal polyhedra

September 14, 2010. 

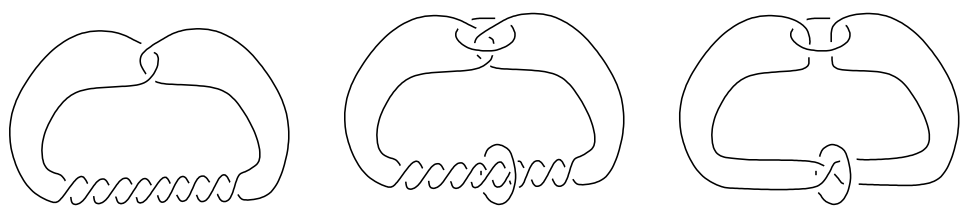

FiguRE 1. Left to right: A link diagram. The augmented link diagram. A diagram of the corresponding fully augmented link.

to improve Lackenby's upper bound on volume. As far as I am aware, this appendix is the first place the polyhedral decomposition appeared in print.

We describe Agol and Thurston's decomposition of fully augmented links into totally geodesic, right angled ideal polyhedra below. Manipulating these polyhedra allows us to determine geometric information on the links, including bounds on volume, cusp shape and cusp area. These have been used to bound exceptional surgeries on knots [7], volumes of knots [6], and cusp shapes [13]. Moreover, the polyhedral decomposition enabled Chesebro, DeBlois, and Wilton to show that fully augmented links satisfy the virtually fibered conjecture [3]. Restricting to an even more geometrically explicit subset of fully augmented links, R. Van der Veen was able to show that such links satisfy a version of the volume conjecture [18]. Thus the geometric properties of these links allow us to prove several interesting facts about the links and their Dehn fillings.

In this paper, we present many of the above geometric results. Most of the results here are not new, with the possible exception of Proposition 3.8, which does not seem to appear elsewhere in the literature. Those results that have appeared before, however, seem to be scattered throughout many different papers. We present them together in this article to give a (more) complete picture of the geometric properties of these links.

While I have made every attempt to refer to the appropriate papers where these results have appeared or have been applied, I may have missed some references that should belong in this expository paper. I apologize for any such omission.

1.1. Acknowledgements. This paper was written following the workshops on hyperbolic geometry, quantum topology, and number theory held at Columbia University in June 2009. We thank the organizers for arranging the workshops, as well as the NSF for its support of the workshops through an FRG grant. In addition, work on this paper was partially funded by NSF grant DMS-0704359.

\section{Polyhedral DeCOMPosition AND CIRCle PACKINGS}

Given a fully augmented link, there is an associated polyhedral decomposition of the link complement into two isometric, totally geodesic, ideal hyperbolic polyhedra, described in Agol and Thurston's appendix to Lackenby's paper [9]. These polyhedra correspond to a circle packing of $S^{2}$, which in turn corresponds to a triangulation of $S^{2}$. All this information can be read off a diagram of a fully augmented link. We review it in this section.

To create the polyhedral decomposition, first note that a fully augmented link contains many geodesic surfaces. 
Lemma 2.1. Let $L$ be a hyperbolic fully augmented link, with no half-twists. The following surfaces are embedded totally geodesic surfaces in the hyperbolic structure on the link complement.

- Each twice-punctured disk bounded by a crossing circle, punctured by two link components lying on the projection plane.

- Each component of the projection plane.

Proof. Any essential 2-punctured disk embedded in a hyperbolic 3-manifold is totally geodesic [1]. We argue that any 2-punctured disk $E$ bounded by a crossing circle must be essential. For suppose there is a compressing disk $D$ with $\partial D \subset E$. Since $\partial D$ is an essential closed curve on $E$, it must encircle one or two punctures of $E$. If only one puncture, then the union of $D$ and the disk in $S^{3}$ bounded by $\partial D$ (with subset $E$ ) forms a sphere in $S^{3}$ met by the link exactly once. This is impossible. Thus $\partial D$ must bound a $2-$ punctured disk $E^{\prime}$ on $E$. Then $E \backslash E^{\prime} \cup D^{\prime}$ is a boundary compressing disk for the crossing circle, contradicting the fact that $L$ is hyperbolic.

Similarly, a boundary compressing disk $D$ for $E$ will have boundary consisting of two arcs, one of which is an essential arc $\alpha$ on $E$ with endpoints on the same component of $\partial E$. For each boundary component of $E$, there is exactly one possibility for $\alpha$ with endpoints on that boundary component, up to isotopy. In each case, $\alpha$ will separate $E$ into two components, at least one containing exactly one puncture. Let $A \subset E$ be the annulus with one boundary component on a puncture of $E$, and the other boundary component consisting of two arcs: one $\operatorname{arc} \alpha$, and the other an $\operatorname{arc}$ on another boundary component of $E$. Now attach $D$ to $A$ along $\alpha$. Because $D$ is disjoint from $E$, this gives an embedded annulus in $S^{3} \backslash L$. The annulus cannot be essential, since $L$ is hyperbolic. Compressing along a compression disk or boundary compression disk for the annulus again would give a boundary compressing disk for $S^{3} \backslash L$. Thus the annulus must be boundary parallel. Since one component of $\partial A$ is a meridian, the other must be as well, and the boundary of $D$ must be isotopic to a closed curve embedded on $E$ encircling both punctures. As before, this is a contradiction.

As for the projection plane, notice that reflection through the projection plane preserves the link complement, fixing the plane pointwise. It is a consequence of Mostow-Prasad rigidity that such a surface must be totally geodesic.

To create the polyhedral decomposition of the link complement $S^{3} \backslash L$, we cut along totally geodesic surfaces.

Proposition 2.2 (Polyhedral decomposition of $\left.S^{3} \backslash L\right)$ ). Let $L$ be a hyperbolic fully augmented link. There is a decomposition of $S^{3} \backslash L$ into two identical totally geodesic polyhedra. In addition, these polyhedra have the following properties.

- Faces of the polyhedra can be checkerboard colored, with shaded faces all triangles corresponding to 2-punctured disks, and white faces corresponding to components of the projection plane.

- Ideal vertices are all 4-valent.

- The dihedral angle at each edge is $\pi / 2$. 

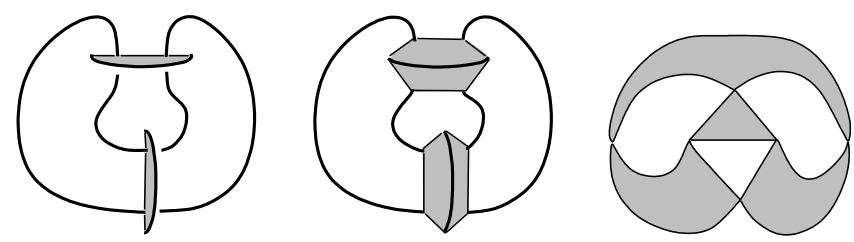

Figure 2. Left to right: Steps 1, 2, and 3.

Proof. First, assume those components of $L$ that are not crossing circles lie flat on the projection plane, i.e. that there are no half-twists. Create the polyhedra by the following procedure, illustrated in Figure 2:

Step 1. Cut $S^{3} \backslash L$ along the projection plane, slicing it into two identical pieces, one above and one below the projection plane. Note this slices each of the 2-punctured disks bounded by a crossing circle in half.

Step 2. For each of the two pieces resulting from Step 1, slice up the middle of the halves of 2-punctured disks, opening half disks out as in Figure 2.

Step 3. Collapse each component of the link to a single ideal vertex.

This decomposes $S^{3} \backslash L$ into two identical polyhedra.

The edges of the polyhedra come from the intersections of the 2-punctured disks with the projection plane. We shade the faces coming from 2-punctured disks and leave the components of the projection plane white. Note that each edge bounds one shaded face and one white face. Hence the polyhedra can be checkerboard colored. Moreover, note that each shaded face meets exactly three edges, hence all are triangles.

Ideal vertices correspond to components of the link after cutting, slicing, and flattening (steps 1 and 2 above). Each component coming from a link component embedded in the projection plane will meet exactly four edges: two for each of the 2-punctured disks in which that link component terminates. Each component coming from a crossing circle will also meet exactly four edges: two for each of the two triangles corresponding to the half of the 2-punctured disk it bounds in each polyhedron. So ideal vertices are 4-valent, as claimed.

Finally, note that reflection in the white faces preserves the link complement. Hence shaded faces must be orthogonal to the white faces, and hence the dihedral angle at each edge is exactly $\pi / 2$.

To obtain $S^{3} \backslash L$ from the polyhedra, reverse the slicing procedure. First glue pairs of shaded triangles across a vertex corresponding to a crossing circle, then glue corresponding white faces in the two polyhedra.

Finally, if $L$ has half-twists, we modify the procedure slightly. Remove all half-twists from the diagram, then repeat Steps 1, 2, and 3 above to obtain two checkerboard colored polyhedra, with properties as claimed in the statement of the lemma. To obtain $S^{3} \backslash L$ from these polyhedra, change the gluing at each half-twist. Rather than glue shaded triangles across their common vertex on a single polyhedron, glue each triangle of one polyhedron to the opposite triangle of the other. See Figure 3. The result is homeomorphic to $S^{3} \backslash L$. 

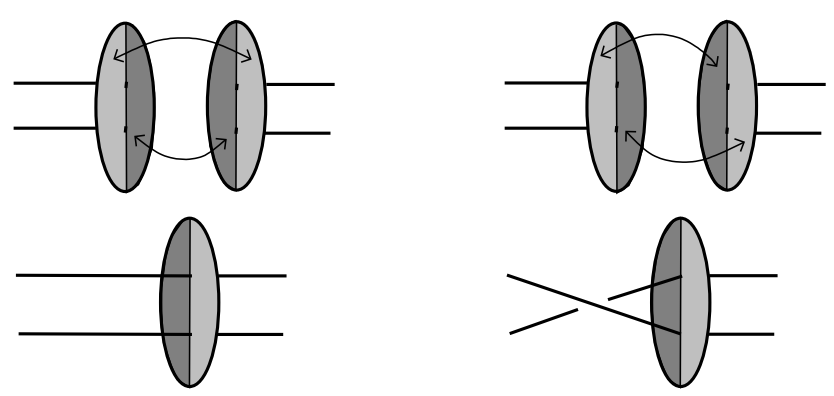

Figure 3. Left: Gluing shaded triangles in same polyhedron across their common vertex gives a crossing circle with no half-twist. Left: Gluing each shaded triangle in one polyhedron to opposite triangle in other polyhedron inserts a half-twist.

Notice that the polyhedra of the polyhedral decomposition of Proposition 2.2 agree for fully augmented links with or without half-twists. Only the gluing of the polyhedra changes when half-twists are present.

By a circle packing, we mean a finite collection of Euclidean circles in $\mathbb{R}^{2}$ or $S^{2}$ which meet only in points of tangency. The nerve of a circle packing is the graph obtained by taking one vertex for each circle, and an edge between two vertices for which the corresponding circles are tangent.

Lemma 2.3. Let $L$ be a hyperbolic fully augmented link. Then the polyhedral decomposition of $S^{3} \backslash L$ corresponds a circle packing on $S^{2}$ whose nerve is a triangulation of $S^{2}$. Moreover, the nerve satisfies the following two properties.

- Each edge of the nerve has distinct endpoints.

- No two vertices are joined by more than one edge.

Proof. The circle packing of the lemma is given by considering the hyperbolic structure on the polyhedra of Proposition 2.2. Each white face is totally geodesic. Hence it extends to the boundary at infinity, $S_{\infty}^{2}$ of $\mathbb{H}^{3}$ to give a Euclidean circle on $S_{\infty}^{2}$. Since none of the white faces overlap, but meet only at ideal vertices, the corresponding circles will meet only in points of tangency corresponding to associated ideal vertices.

The fact that the nerve of the circle packing is a triangulation follows from the fact that the shaded faces of the polyhedra are all triangles. White faces meet at ideal vertices, so corresponding to each ideal vertex is an edge of the nerve. Two triangular shaded faces also meet at each ideal vertex. These group ideal vertices into triples. Corresponding edges of the nerve form a triangle. See Figure 4.

Finally, notice that since the circles of our circle packing are geometric circles on $S_{\infty}^{2}$, a circle cannot be tangent to itself, so each edge has distinct endpoints. Moreover, if two circles are tangent, then they may only be tangent in a single point. So no two vertices are joined by more than one edge.

The circle packing of Lemma 2.3 is obtained by extending white faces to the boundary at infinity of $\mathbb{H}^{3}$. Notice that the dual circle packing is given by extending shaded faces to the 

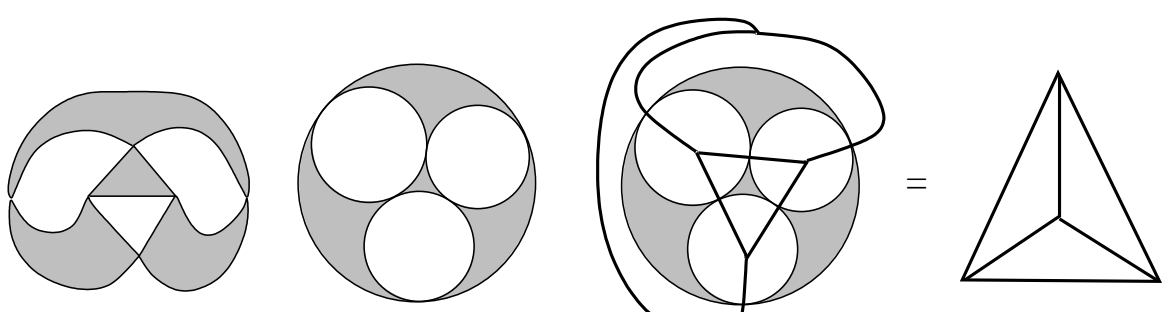

FiguRE 4. An example of a circle packing and its nerve.

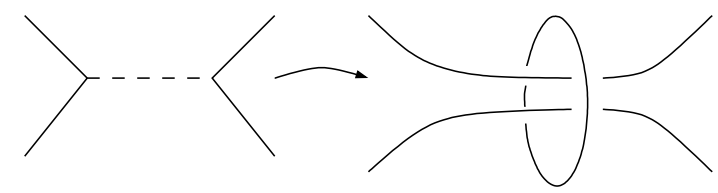

FiguRE 5. Each red edge (shown here dashed) is replaced by a crossing circle.

boundary at infinity of $\mathbb{H}^{3}$. To obtain each polyhedron, take the intersection of appropriate half spaces in $\mathbb{H}^{3}$ with boundary the hemispheres corresponding to these circles.

There is a converse to Lemma 2.3.

Lemma 2.4. Let $\gamma$ be a triangulation of $S^{2}$ such that each edge has distinct ends and no two vertices are joined by more than one edge. Choose a collection of edges of $\gamma$ and paint them red, such that each triangle of $\gamma$ meets exactly one red edge. Then associated to this painted graph is a hyperbolic fully augmented link, the nerve of which is $\gamma$.

Proof. By a corollary of Andreev's theorem noted by W. Thurston [16, Chapter 13], associated to $\gamma$ is a circle packing, unique up to Möbius transformation, whose nerve is isotopic to $\gamma$. The circles in the circle packing extend to give hemispheres in $\mathbb{H}^{3}$. Circles dual to our circle packing also extend to give hemispheres in $\mathbb{H}^{3}$. The intersection of half-spaces in $\mathbb{H}^{3}$ bounded by all these hemispheres, lying between the hemispheres gives an ideal polyhedron in $\mathbb{H}^{3}$.

We may construct the complement of a hyperbolic fully augmented link by gluing together faces of this polyhedron and an identical copy of this polyhedron. In particular, red edges of $\gamma$ will correspond to crossing circles. First glue together triangular shaded faces across each of these vertices in each polyhedron. Then glue corresponding white faces across the two polyhedra.

We need to ensure this actually is homeomorphic to the complement of a fully augmented link. To see the diagram of the fully augmented link, let $\Gamma$ be the graph dual to $\gamma$. It is a trivalent graph on $S^{2}$ with one of every three edges colored red. Replace each red edge by a crossing circle, as in Figure 5. Then one easily checks that the circle packing associated with this augmented link agrees with that given by Lemma 2.3.

Figure 5 gives us a combinatorial way of associating a triangulation of $S^{2}$ with the diagram of a fully augmented link without stepping through the polyhedral decomposition. Namely, 

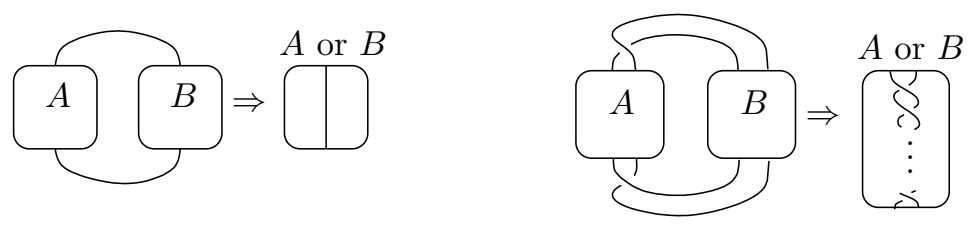

Figure 6. Left: A prime diagram. Right: A twist reduced diagram.

given any augmented link, replace crossing circles (and any half-twists) with red edges, connecting the strands entering into the crossing circle into a trivalent vertex before and after the crossing circle, as in the figure. This gives a trivalent planar graph on $S^{2}$. Its dual graph, a triangulation of $S^{2}$, is the nerve of the circle packing of Lemma 2.3.

A choice of red edge for each triangle above is a choice of dimer for the graph $\gamma$. Thus enumerating all (not necessarily distinct) fully augmented links with a given number of crossing circles amounts to enumerating triangulations of $S^{2}$ with a fixed number of triangles, along with a choice of dimer for each triangulation. Additionally, there is the choice at each red edge as to whether to insert a half-twist or not.

The hyperbolicity of a fully augmented link $L$ obtained by augmenting the diagram of a link $K$ is known to be related to the diagram of $K$, as well. Recall that a diagram is prime if whenever a simple closed curve on the projection plane intersects the diagram in exactly two points, the curve bounds a portion of the projection plane containing no crossings. A diagram is twist reduced if whenever a simple closed curve on the projection plane intersects the diagram in four points, adjacent to two crossings, the curve bounds a region of the projection plane containing a (possibly empty) twist region. See Figure 6.

The following theorem follows easily from work of Adams [2].

Theorem 2.5. A fully augmented link is hyperbolic if and only if the associated knot or link diagram is nonsplittable, prime, twist reduced, with at least two twist regions.

The more difficult direction can also be shown using Andreev's theorem, which was done in [13, Theorem 6.1]. As for the other direction, if the diagram is not prime or not twist reduced the corresponding fully augmented link will contain an obvious incompressible annulus. One twist region (or fewer) gives a Seifert fibered augmented link. We omit the full details here.

\section{Geometric properties of Fully Augmented Links}

3.1. Cusp shape. Restriction of the hyperbolic metric on the complement of a fully agumented link to a horospherical torus about each link component gives a Euclidean structure on the torus [17]. For more general hyperbolic manifolds, it is often difficult to determine the Euclidean structure about a cusp. For fully augmented links, however, the cusp shape can be read off of the circle packing of Lemma 2.3 and the gluing of the polyhedra.

Lemma 3.1. Any cusp of a fully augmented link is tiled by rectangles, each determined by a circle packing corresponding to a vertex of the ideal polyhedra of Proposition 2.2. Moreover, the circle packing for any such vertex always consists of two parallel white lines, both tangent to a pair of white circles as in Figure 7, with all additional circles interior to these four. 

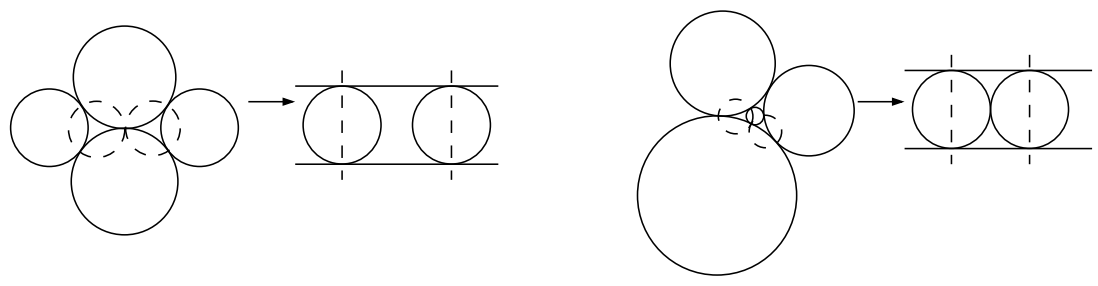

FiguRE 7 . Result of taking any ideal vertex to infinity is a rectangle.

Proof. Any cusp of a fully augmented link will correspond to a collection of ideal vertices in the polyhedral decomposition. Proposition 2.2 implies that ideal vertices are rectangles: each ideal vertex is 4 -valent and dihedral angles are $\pi / 2$. To obtain the circle packing, consider points of tangency of circles in the circle packing of Lemma 2.3. Select one of these points of tangency, call it $p$. The circle packing lies on the boundary at infinity of $\mathbb{H}^{3}$, i.e. on the Riemann sphere $S_{\infty}^{2}=\mathbb{C} \cup\{\infty\}$. Therefore there exists a Möbius transformation, an isometry of $\mathbb{H}^{3}$, taking the point $p$ of tangency of the two circles to the point $\infty$ in $\mathbb{C} \cup\{\infty\}$.

Consider the effect of this Möbius transformation on the circle packing. The two circles tangent at $p$ will go to parallel lines through $\infty$. Since the nerve of the circle packing is a triangulation, on either side of $p$ there is a closest circle tangent to both circles mapped to parallel lines. Each of these two circles will be mapped to circles tangent to both parallel lines, as in Figure 7. All other circles will be mapped under the Möbius transformation to circles lying between these four.

In Figure 8, we show several examples of fully augmented links and the associated "rectangular" circle packing, seen with an ideal vertex at infinity.

We remark that many additional beautiful examples of fully augmented links appear in Chesebro, DeBlois, and Wilton [3, Section 7]. They show the examples of fully augmented links together with a trivalent graph they call the crushtacean of the link. In our terminology, this crushtacean is exactly the dual graph to the nerve of the circle packing associated with fully the augmented link.

To obtain the shapes of the cusps of augmented links, we need to look at the tilings of cusps by the rectangles of Proposition 2.2. This is done by walking through the gluing of polyhedra, forming fundamental domains for the cusps, keeping track of curves corresponding to meridians and longitudes. This is done very carefully in [7]. We reproduce the argument here for crossing circles, and leave shapes of other cusps as an exercise for the reader. The following is part of Lemma 2.3 of [7].

Proposition 3.2. Let $C$ be a cusp corresponding to a crossing circle in a hyperbolic fully augmented link. Then the following hold:

(1) A fundamental region for $C$ consists of two rectangles coming from vertices of the polyhedra of Proposition 2.2.

(2) A longitude of $C$ is parallel to the curve given by a shaded face intersected with the cusp boundary, and it intersects white faces twice. See Figure 9.

(3) In the case $C$ corresponds to a crossing circle with no half-twist, a meridian is parallel to the curve given by a white face intersected with the cusp boundary. 

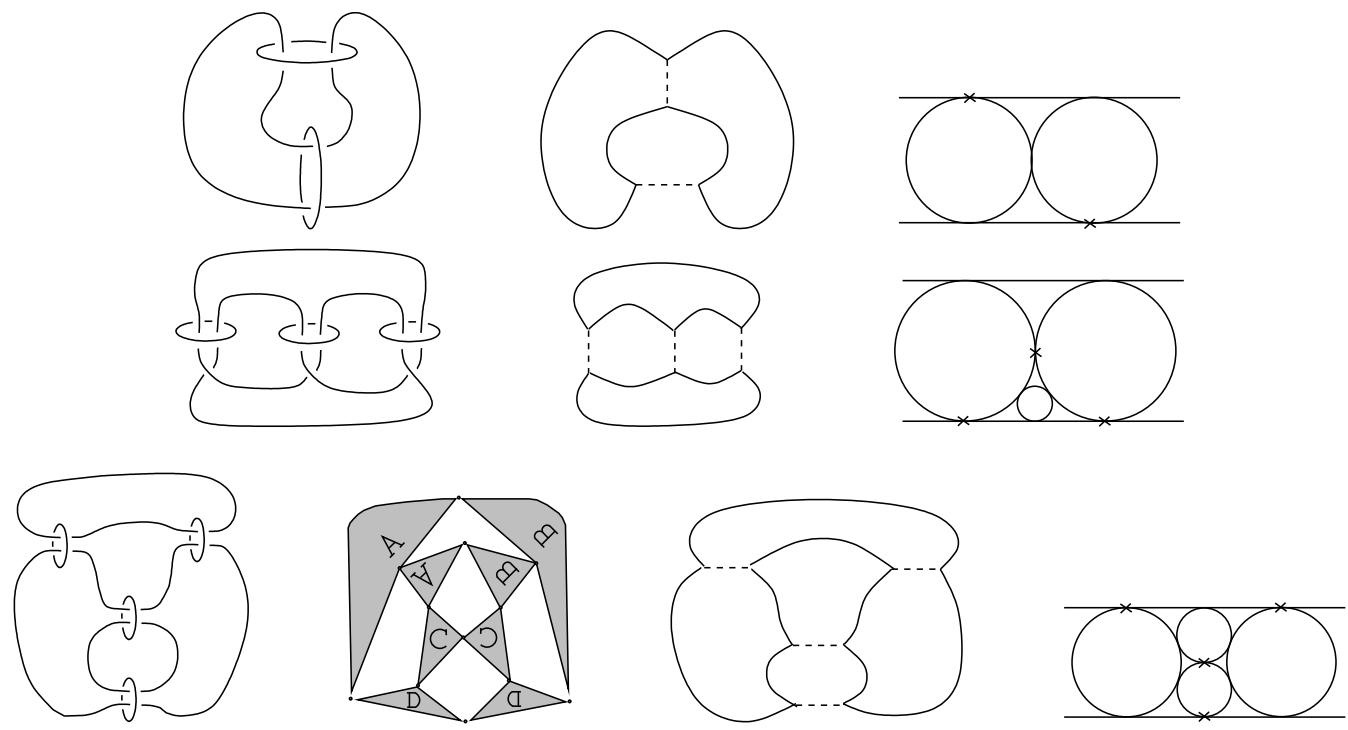

Figure 8. Examples of fully augmented links with 2, 3, and 4 crossing circles, their associated trivalent graphs, and their circle packings. For the third example, combinatorics of polyhedral decomposition are also shown.

(4) If C corresponds to a crossing circle with a half-twist, then a meridian connects the corner of one of the rectangles of Figure 7 to the opposite corner.

Proof. The result is obtained by analyzing the gluing across the rectangles of Figure 7. A rectangle corresponding to a crossing circle cusp is shown for an example on the left in Figure 9. Note that there are exactly two such rectangles, one on one of the two polyhedra in the decomposition, and one on the other. The reflection of $S^{3} \backslash L$ in the white faces of the polyhedra glues these two rectangles together along a side corresponding to the intersection of a white face with the cusp. These are the only rectangles corresponding to this cusp, so part (1) holds.

From the diagram of the fully augmented link, we see that a longitude of a crossing circle is parallel to the shaded disk bounded by the crossing circle. In the polyhedral decomposition, this shaded disk becomes shaded faces. The intersection of the shaded disk with the boundary of the cusp therefore projects to the longitude. Since the two rectangles of part (1) are glued along white faces, a longitude must run over both rectangles and intersect white faces twice, for example as in the middle in Figure 9. This gives part (2).

When there are no half-twists, a meridian can be seen in the diagram of the fully augmented link. It runs along the projection plane, which is one of the totally geodesic faces of Lemma 2.1, and becomes a white face of the polyhedral decomposition. Thus the intersection of a white face with the cusp boundary projects to a meridian. Note its endpoints are on shaded faces which glue to each other, hence it steps along just one rectangle of Figure 7, as shown for example in Figure 9. This gives part (3). 

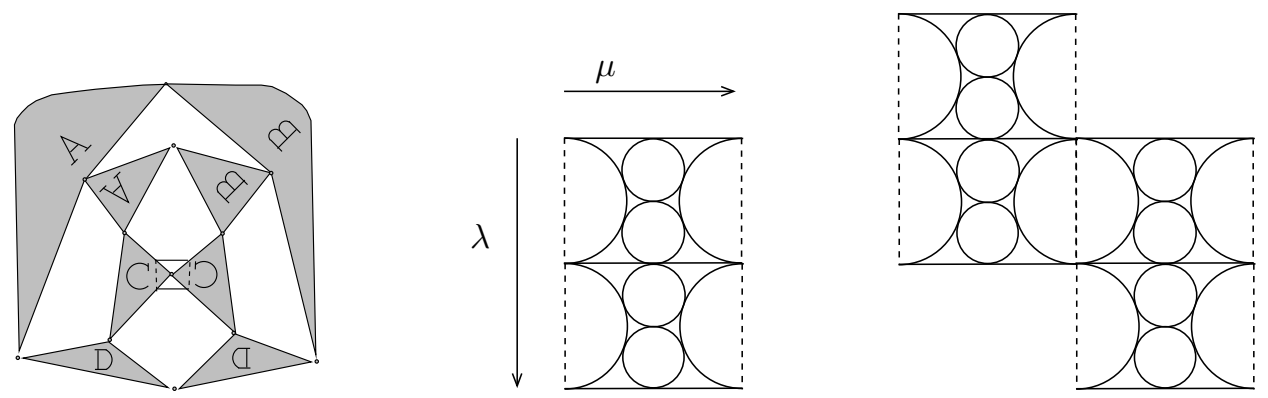

Figure 9. Left: Ideal vertex corresponding to crossing circle. Middle: Fundamental region of associated cusp of $S^{3} \backslash L$. Without half-twists, $\lambda$ is a longitude, $\mu$ a meridian. Right: Half-twists cause shearing.

Finally, if $C$ corresponds to a crossing circle with a half-twist, then a shaded triangle on one polyhedron is glued to a shaded triangle opposite the vertex corresponding to $C$ on the other polyhedron. We see this as a shear in the universal cover, as in the right in Figure 9. The meridian travels a step along a white side, followed by a step along a shaded side (in a direction corresponding to the direction of the half-twist). This gives part (4).

3.2. Canonical polyhedral decomposition. In this section, we show that edges of the polyhedra obtained in Proposition 2.2 are actually canonical, as defined by Epstein and Penner [5]. That is, these edges are geometric duals to faces of a Ford domain. Recall that when a 3-manifold has more than one cusp, as in the case of fully augmented link complements, a Ford domain corresponds to a choice of horoball neighborhoods about all cusps. We will see there is a choice of horoball neighborhoods with disjoint interiors such that the horoball neighborhoods are tangent across every edge of the polyhedra of Proposition 2.2. It follows that each edge is in the geometric dual of this Ford domain.

Definition 3.3. Given any edge $e$ of a hyperbolic ideal triangle $T$, define the midpoint of $e$ to be the point on $e$ where a geodesic from the vertex of $T$ opposite $e$ meets $e$ at a right angle.

In the complement of a fully augmented link, shaded faces form triangles. Thus for every edge of every shaded face, we may find a midpoint. Two shaded faces are glued to each other by a reflection through a white face. Since angles are preserved under reflection, the midpoint of an edge on one shaded face will agree with the midpoint of the same edge in an adjacent shaded face. Thus each edge of the polyhedral decomposition of a fully augmented link has a well-defined midpoint.

The following is Theorem 3.8 of [7].

Theorem 3.4. Let $L$ be a fully augmented link. Then there exists a horoball expansion about the cusps of $S^{3} \backslash L$ such that the midpoint of every edge is a point of tangency of horospherical tori.

Proof. Let $P$ be one of the (identical) ideal polyhedra in the decomposition of $S^{3} \backslash L$. First, lift $P$ to $\mathbb{H}^{3}$ so that the (Euclidean) width of the rectangle of Figure 7 is exactly 1 . That 
is, the two circles forming sides of the rectangle of Figure 7 have diameter 1 . There are exactly four edges meeting the ideal vertex at infinity. Note the midpoint of each of them is at (Euclidean) height 1 in $\mathbb{H}^{3}$. Thus a horizontal plane of (Euclidean) height 1 in $\mathbb{H}^{3}$ is a horosphere meeting all the midpoints of edges through the vertex at infinity.

Suppose we cannot expand a horoball neighborhood about all cusps to the midpoints of the edges. Expand cusps as much as possible, without expanding any cusp beyond the midpoints of the adjacent edges. In the universal cover, we will see a tiling of $\mathbb{H}^{3}$ by copies of $P$ which we may assume have Euclidean width 1 . By assumption, we may apply an isometry so that the horoball about infinity cannot be expanded to (Euclidean) height 1. Thus there is a horoball $H$ of diameter $h>1$ tangent to the horoball about infinity, which projects to some embedded cusp of $S^{3} \backslash L$ which has not been expanded beyond midpoints of edges. Let $z \in \mathbb{C}$ be the center of the horoball $H$ of diameter $h>1$.

First, notice that $z$ cannot lie strictly between the two white vertical planes of the rectangles of Lemma 3.1, as follows. If so, since the vertical white planes are (Euclidean) distance 1 apart, and $H$ has (Euclidean) diameter $h>1, H$ must intersect at least one of the two vertical white planes. Since a reflection in the white planes preserves the link complement, the isometry given by reflection in the plane meeting $H$ descends to an isometry of $S^{3} \backslash L$. But under this isometry, $H$ is taken to a horoball intersecting $H$. This contradicts the fact that our horoball was embedded. Thus $z$ must lie on one of the vertical white planes forming top and bottom sides of the rectangle of Figure 7, and the reflection takes $H$ to itself.

Next, notice that $z$ cannot lie at the endpoint of an edge of the polyhedral decomposition running down from infinity, for if so, $H$ contains the midpoint of this edge in its interior. This is a contradiction: we assumed that $H$ was not expanded beyond any midpoints.

Finally, we prove that $z$ cannot lie in the interior of one of the vertical white faces, disjoint from an edge through infinity. For if so, say $z$ lies on the vertical white plane $V$, since $z$ is an ideal vertex of a copy of the polyhedron $P$, there exists a white plane in $\mathbb{H}^{3}$ tangent to $V$ at the point $z$, so that $V$ and this white plane are both boundary faces of $P$. The white plane has boundary a circle $C$ on $\mathbb{C}$. Consider the projection of $C$ to $V$. That is, consider the set of geodesics through points of $C$ meeting $V$ at right angles. The endpoints of these arcs on $V$ define a (Euclidean) circle $C^{\prime}$ of the same diameter as $C$, tangent to $z$. Since $C$ lies between two vertical planes, it has diameter less than 1 . Thus $C^{\prime}$ is contained in the interior of $H$.

Now apply an isometry taking $z$ to infinity, taking $V$ to itself, and taking the plane with boundary $C$ to a vertical plane of distance 1 from $V$. The circle $C^{\prime}$ on the vertical plane is taken to a horizontal line on $V$ of height exactly 1, which must still lie in the interior of the image of $H$. But this is impossible: we assume that $H$ was not expanded beyond the midpoints of the edges meeting it. This final contradiction finishes the proof.

Corollary 3.5. The edges of the ideal polyhedra of Proposition 2.2 are canonical edges for the link complement, in the sense of Epstein and Penner [5].

Note that there may be additional canonical edges in the canonical polyhedral decomposition. 


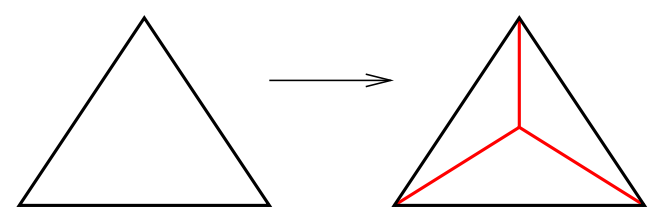

Figure 10. Central subdivision.

3.3. Volume bounds for fully augmented links. We discussed volumes of fully augmented links in [6]. The following is Proposition 3.1 of that paper.

Proposition 3.6. Let $L$ be a hyperbolic fully augmented link with c crossing circles. Then its volume is at least $2 v_{8}(c-1)$, where $v_{8}=3.66386 \ldots$ is the volume of a regular ideal octahedron. Moreover, the volume is exactly $2 v_{8}(c-1)$ if and only if $S^{3} \backslash L$ decomposes into regular ideal octahedra.

Proof. By work of Adams [1], the volume of the complement of $L$ agrees with that of the augmented link with no half-twists, so we will assume $L$ has no half-twists. Cut $S^{3} \backslash L$ along the projection plane, dividing it into two isometric hyperbolic manifolds with totally geodesic boundary, where the isometry is given by reflection in the projection plane.

Miyamoto showed that if $N$ is a hyperbolic 3-manifold with totally geodesic boundary, then $\operatorname{vol}(N) \geq-v_{8} \chi(N)$ [10], with equality exactly when $N$ decomposes into regular ideal octahedra. In our case, each cut half of $S^{3} \backslash L$ is a ball with a tube removed for each crossing circle, hence it is a genus $c$ handlebody, and has Euler characteristic $1-c$. The result now follows.

Definition 3.7. Given a triangle $T$, define the central subdivision of $T$ to be the subdivision obtained by inserting a vertex in the center of $T$, then adding three edges running from the new vertex to one of the three vertices of $T$. See Figure 10.

The following proposition gives all examples of fully augmented links for which the polyhedra of Proposition 2.2 decompose into regular ideal octahedra. Hence for these links, the estimate of Proposition 3.6 is sharp.

Proposition 3.8. Let $L$ be a fully augmented link with polyhedral decomposition into two polyhedra isometric to $P$, and let $N$ be the nerve associated with the circle packing of $L$. Then $P$ is obtained by gluing regular ideal octahedra if and only if $N$ is obtained by central subdivision of the complete graph on four vertices. In this case, there are $c-1$ such octahedra, where $c$ is the number of crossing circles in the diagram of $L$.

Proof. Suppose the polyhedral decomposition of $L$ gives two polyhedra which are obtained by gluing regular ideal octahedra. Apply a Möbius transformation taking a vertex of one of the polyhedra to infinity. This vertex is an ideal vertex of at least one of the regular octahedra. Under the Möbius transformation, any octahedron with vertex taken to infinity will give rise to a collection of circles of the circle packing of the form in Figure 11, on the left.

If multiple octahedra share the vertex at infinity, then we claim they must be glued together in a linear manner as shown on the right of Figure 11. This can be seen by 

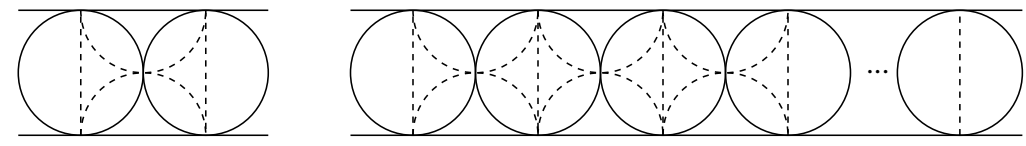

FiguRE 11. Left: Top down view of a regular ideal octahedron. Right: such octahedra sharing the ideal vertex at infinity must be glued in a linear manner, as shown. (Dashed lines show location of dual circles, giving shaded faces.)
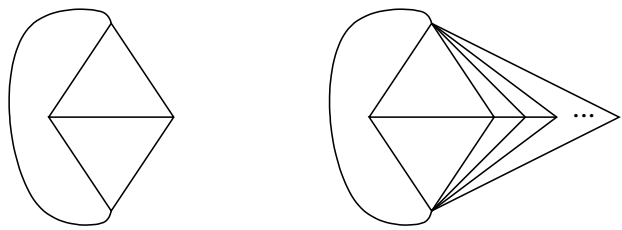

FiguRE 12. Left: Nerve of circles of octahedron is complete graph on four vertices. Right: For multiple octahedra glued at vertex at infinity, nerve is central subdivision of graph on the left.

an inductive argument. Given a single octahedron, a second octahedron meeting at the vertex at infinity will be attached to the first either along a white face or one of the two shaded faces. If it is attached to a white face, then the two shaded faces on the ends become quadrilaterals, contradicting Proposition 2.2. By induction, the $n$-th octahedron must be attached along one of the shaded faces as well, else again we have a contradiction to Proposition 2.2.

Consider the portion of the nerve of the circle packing corresponding to the circles of octahedra sharing this vertex at infinity. If we have just one ideal octahedron, the nerve is the complete graph on four vertices, as shown on the left of Figure 12. If there are more octahedra, the result is a central subdivision of the complete graph on four vertices, subdividing the triangle enclosing the point at infinity, as on the right of Figure 12.

The above holds for any ideal vertex. When we move a different ideal vertex to infinity, we apply a Möbius transformation. This will change the nerve by moving a new edge to infinity. (In Figure 12 we pushed this edge off infinity slightly to obtain the curved edge in that figure.) While the vertices and edges of the nerve may move around, each triangle before the Möbius transformation corresponds to a triangle after, with vertices and edges the images of vertices and edges before. Thus the triangle containing infinity in the nerve before the Möbius transformation, which we saw was subdivided by central subdivision once for every octahedron meeting infinity, will be mapped to a triangle which has been subdivided by central subdivision. Thus the entire nerve is obtained by central subdivision of the complete graph on four vertices.

Now suppose the nerve of the fully augmented link $L$ is obtained by successive central subdivision of the nerve of the connected graph on four vertices. We induct on the number of times we must subdivide to obtain the nerve of $L$. If 0 times, then the nerve of $L$ is the 

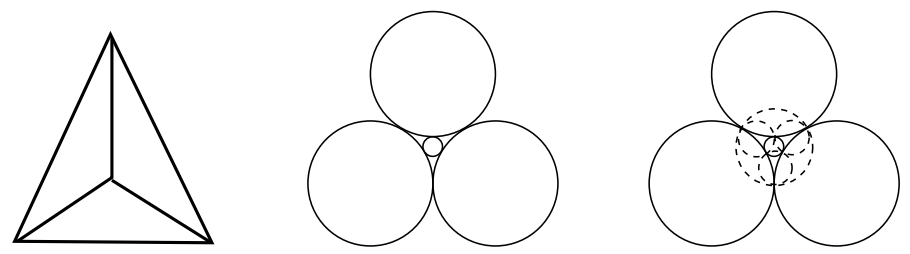

Figure 13. Left: Subdivide a triangle in the nerve. Middle: add a circle to the corresponding circle packing. Right: Attach a polyhedron bounded by the hemispheres of these circles, and their duals.

graph shown on the left of Figure 12, and the corresponding polyhedron is a single regular ideal octahedron shown on the left of Figure 11.

Next suppose that we have subdivided $n$ times, each time attaching a new regular ideal octahedron to the previous polyhedron. At the $(n+1)$-st subdivision, we add a vertex and three edges to some triangle of the nerve, as on the left of Figure 13. This corresponds to adding a circle to a triangle of the circle packing, as in the middle of Figure 13. In the 3-dimensional polyhedron, we attach a polyhedron with faces bounded by circles as shown on the right of Figure 13. In particular, note that if we take any of the points of tangency of circles to infinity in that Figure, we obtain exactly the circles on the left of Figure 11. Thus this polyhedron is exactly a regular ideal octahedron.

Finally, we relate the number of octahedra to the number of crossing circles $c$. Suppose the polyhedron of Proposition 2.2 is obtained by gluing $n$ regular ideal octahedra. Then the nerve is formed by central subdivision of the complete graph on four vertices. From the proof above, the first octahedron corresponds to that complete graph on four vertices, containing 6 edges, and then each successive subdivision, adding 3 edges, gives exactly one more octahedron. Thus the number of edges $E$ in the nerve is $E=3 n+3$.

On the other hand, the nerve is a triangulation of $S^{2}$. For each triangle, exactly one edge is painted red, i.e. corresponds to an ideal vertex associated with a crossing circle. A single red edge borders two triangles. Thus the total number of red edges is equal to half the total number of triangles, $T$, in the nerve. Since each red edge corresponds to a distince crossing circle, we have $c=T / 2$. Because the nerve is a triangulation of $S^{2}, 3 T=2 E=6 n+6$. Hence $c=n+1$, or there are exactly $c-1$ octahedra, as claimed.

\section{RESTRICTING AND EXTENDING RESUlts}

In this section, we study two examples of classes of links related to fully augmented links. The first class is obtained by restricting to a subset of fully augmented links, obtaining a class of links even more geometrically explicit. The second class is obtained by extending to a class of links containing fully augmented links, losing some of the geometric structure as we relax the definitions.

4.1. Octahedral fully augmented links. The geometry of fully augmented links is completely determined by a circle packing, which can be computed from a nerve, for example 
in simple cases by K. Stevenson's program CirclePack [4]. However, for extremely complicated nerves or families of nerves it may be more difficult to obtain circle packings. Also, additional geometric estimates such as volume may be harder to obtain exactly.

To remedy this problem, we may restrict to a subclass of fully augmented links: those whose polyhedra are a union of regular ideal octahedra. For these links, which we will call octahedral augmented links, the volume is given exactly by Proposition 3.6. The circle packing can be constructed by hand from the nerve (provided your pencil tip is sharp enough). The rectangles of Lemma 3.1 are all squares, or strings of squares as in Figure 11. The geometry is completely explicit. Indeed, among these links are many links for which the volume conjecture is known, due to work of R. Van der Veen [18].

4.2. Generalized fully augmented links. We may generalize the class of fully augmented links by relaxing conditions of their definition. In a generalized augmented link, we allow crossing circles to encircle more than two strands per twist region. For these links, we lose the triangulation and circle packing. Results on hyperbolicity and cusp shapes are not as clean $[12,14]$. However, there is still a reflection in the projection plane, giving a totally geodesic surface when these links are hyperbolic. The existence of this surface and the reflection allows us to obtain some information on volumes and geometry of these links. These are explored in [11] and in [14].

\section{REFERENCES}

[1] Colin C. Adams, Thrice-punctured spheres in hyperbolic 3-manifolds, Trans. Amer. Math. Soc. 287 (1985), no. 2, 645-656.

[2] _ Augmented alternating link complements are hyperbolic, Low-dimensional topology and Kleinian groups (Coventry/Durham, 1984), London Math. Soc. Lecture Note Ser., vol. 112, Cambridge Univ. Press, Cambridge, 1986, pp. 115-130.

[3] Eric Chesebro, Jason DeBlois, and Henry Wilton, Some virtually special hyperbolic 3-manifold groups, arXiv:0903.5288.

[4] Tomasz Dubejko and Kenneth Stephenson, Circle packing: experiments in discrete analytic function theory, Experiment. Math. 4 (1995), no. 4, 307-348.

[5] D. B. A. Epstein and R. C. Penner, Euclidean decompositions of noncompact hyperbolic manifolds, J. Differential Geom. 27 (1988), no. 1, 67-80.

[6] David Futer, Efstratia Kalfagianni, and Jessica S. Purcell, Dehn filling, volume, and the Jones polynomial, J. Differential Geom. 78 (2008), no. 3, 429-464.

[7] David Futer and Jessica S. Purcell, Links with no exceptional surgeries, Comment. Math. Helv. 82 (2007), no. 3, 629-664.

[8] Marc Lackenby, Word hyperbolic Dehn surgery, Invent. Math. 140 (2000), no. 2, 243-282.

[9] _ The volume of hyperbolic alternating link complements, Proc. London Math. Soc. (3) 88 (2004), no. 1, 204-224, With an appendix by Ian Agol and Dylan Thurston.

[10] Yosuke Miyamoto, Volumes of hyperbolic manifolds with geodesic boundary, Topology 33 (1994), no. 4, 613-629.

[11] Jessica S. Purcell, Hyperbolic geometry of multiply twisted knots, arXiv:0709.2919.

[12] _ On multiply twisted knots that are Seifert fibered or toroidal, arXiv:0906.4575.

[13] _ Cusp shapes under cone deformation, J. Differential Geom. 80 (2008), no. 3, 453-500.

[14] _ Slope lengths and generalized augmented links, Comm. Anal. Geom. 16 (2008), no. 4, 883-905.

[15] Dale Rolfsen, Knots and links, Publish or Perish Inc., Berkeley, Calif., 1976, Mathematics Lecture Series, No. 7.

[16] William P. Thurston, The geometry and topology of three-manifolds, Princeton Univ. Math. Dept. Notes, 1979. 
[17] _ Three-dimensional geometry and topology. Vol. 1, Princeton Mathematical Series, vol. 35, Princeton University Press, Princeton, NJ, 1997, Edited by Silvio Levy.

[18] Roland van der Veen, The volume conjecture for augmented knotted trivalent graphs, Algebr. Geom. Topol. 9 (2009), no. 2, 691-722.

Department of Mathematics, Brigham Young University, Provo, UT 84602

E-mail address: jpurcell@math.byu.edu 